\title{
Incorporating Artificial Skin Signals in the Constraint-based Reactive Control of Human-Robot Collaborative Manipulation Tasks
}

\author{
Cristian Alejandro Vergara Perico*,1, Gianni Borghesan ${ }^{1}$, Erwin Aertbeliën ${ }^{1}$, Joris De Schutter ${ }^{1}$
}

\begin{abstract}
-
Purpose: The aim of this work is to develop a control strategy for human-robot collaborative manipulation task that can deal with proximity signals from 373 interconnected cells of an artificial skin.

Methodology: The robot and the operator accomplish an industrial task while interacting in a shared workspace. The robot controller detects and avoids collisions based on the information from the artificial skin. Conflicting constraints can be handled by prioritizing between hard and soft constraints or by weighing the different constraints.

Findings: Weak soft constraints (low weight) are specified to command the robot to move along a nominal path with constant velocity. Stronger soft constraints (higher weight) prevent collisions by means of either moving the end effector backwards along the path or circumventing an obstacle. The proposed approach is validated experimentally.

Originality: A discrete optimization algorithm activates an a-priori selected maximum number of cells. The algorithm selects the appropriate distribution based on the amplitude of each signal and the spatial distribution of the proximity measurements. A second contribution is the specification of a human-robot collaborative application as an optimization problem using eTaSL (expression graph task specification language), which provides reactive control.
\end{abstract}

Keywords - Human-Robot Interaction, Constraint-Based Task Specification and Control, Artificial Robotic Skin.

\section{INTRODUCTION}

More and more robots are not only deployed in large industries such as the car industry, but also in small and medium enterprises (SMEs). To be successful in SMEs, the deployment of a robot application needs to be faster and cheaper. Conditioning the environment or the product input flow to a specific robot application by building fences and using conveyor belts with accurately positioned work pieces has become unaffordable. The Factory-in-a day project (Factory in a Day 2014) proposed an extended collaboration between human and robots, in which robots perform tasks that require high power and/or a minor level of decision making, while humans perform tasks where there is a need for a high level of dexterity and the ability to discern between non-trivial options.

This calls for new sensors and control strategies to enable safe cooperation in shared workspaces. One of the most

\footnotetext{
* Corresponding author, cristian.vergara@kuleuven.be

${ }^{1}$ Department of Mechanical Engineering, KU Leuven, core Lab Flanders Make, Belgium
}

common strategy used nowadays in industry, and already incorporated in the new generation of collaborative robots such as UR10, KUKA LWR, ABB Yumi (Bélanger-Barrete 2015), consists of detecting collisions by means of monitoring the currents fed to motors. When these motor currents are not consistent with a dynamic model of the robot and its load, a stop signal is issued. In a related approach, Haddadin et al. (2018) used a disturbance observer to detect collision; Albini et al. (2017) developed an artificial skin that provides only tactile feedback enabling the controller to detect collisions. These methods have in common that they are able to react only after the collision has occurred.

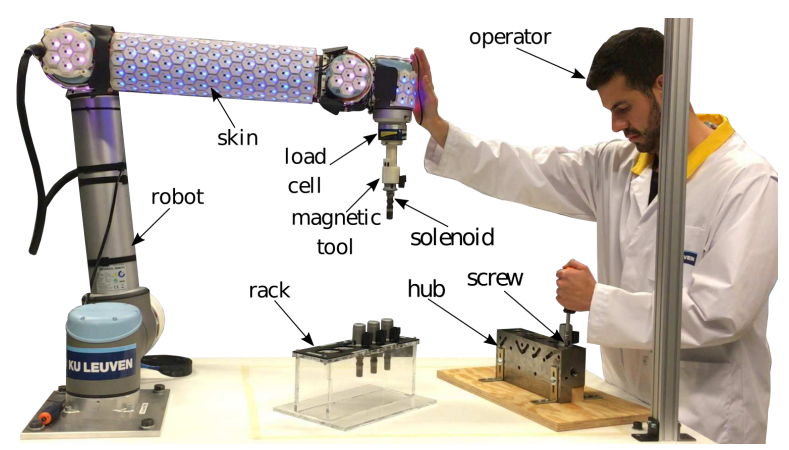

Fig. 1. Human-robot collaborative workstation in an industrial use case. The operator interrupts the robot motion while accomplishing his task.

Zanchettin et al. (2016) used a more proactive approach based on the perception of the scene through a point cloud reconstruction acquired with a Kinect sensor and human motion tracking using OpenNI drivers. They proposed to decrease the velocity of the robot joints in function of the minimum distance between the robot and the obstacle (e.g. human arms); once the measurement is under a lower threshold the robot maintains its position, and the robot only restarts when the distance increases above the threshold. A similar approach of velocity reduction was tested in simulation by (Shin et al. 2018). The velocity reduction proposed by both methods occurs even when objects are close enough but not interfering with the robot motion. As a result, unnecessary stops are induced.

Dumonteil et al. (2015) proposed a method to command the robot to move between two points with the capability of circumventing obstacles. They continuously monitor the workspace with a Kinect sensor. The collision detection is 
done by the Kineo module (KDC), which takes as an input an OctoMap representation (Hornung et al. 2013) of a filtered point cloud. Once a surface section of an obstacle along a nominal trajectory is reconstructed, the KineoWorks software re-plans a new trajectory to circumvent the reconstructed surface. As the method is based in a scene representation using a Kinect sensor, it is very sensitive to a scene occlusions. Zeestraten et al. (2016) tested a method in simulation, which enables a robot to adapt to human motions. However, their algorithm needs prior human demonstrations to generate new trajectories.

Luo et al. (2017) described a control strategy that uses partial observations of human reaching motions to predict the remainder of the trajectories. Their unsupervised on-line learning approach uses Gaussian mixture models, requires no off-line training or manual labeling, and can build prediction models for the reaching motions of the human operator. This information allows the system to avoid possible collisions by either stopping the robot motions or re-planning a trajectory towards a new target. The poses of the human are captured by placing markers on the human limbs and tracking them with a VICON tracking system. In a similar approach Sadrfaridpour \& Wang (2018) developed a trajectory selection algorithm based on human intention prediction, and included a method to measure and include human trust in their model. As in (Luo et al. 2017) human motions are tracked by using a VICON system, which can be sensitive to occlusions when the environment is not conditioned enough.

Similarly as in this work, Buizza Avanzini et al. (2014) developed a human robot collaboration strategy based on the perception of a human in the robot workspace using proximity sensors. Twenty sensors placed in the robot body feed a controller which considers three scenarios based on the intensity of the proximity signal: (i) change the robot configuration without affecting the task performance when the signals report a low value i.e. large distance; (ii) alter the robotic task when the value of the proximity signals is medium; (iii) and stop the robot motion when the signals report a high value i.e. small distance. Therefore, this strategy does not allow to have a close human robot collaboration behavior, and it requires sensor systems that are able to detect objects from far away.

In this paper, the presence of an obstacle that appears in the scene is sensed by proximity sensors embedded in an artificial robotic skin (Mittendorfer et al. 2015); This skin covers the last links of an industrial robot UR10 and contains 373 interconnected cells (see Fig. 1).

The robot behavior and its interaction with the operator is specified using a constraint-based reactive control strategy. Several constraint-based frameworks are found in literature, such as iTaSC (De Schutter et al. 2007) stackof-tasks (Mansard et al. 2009) and expression-based Task Specification Language (eTaSL) (Aertbeliën \& De Schutter 2014, Aertbeliën 2016). These modular approaches are able to combine constraints related to the task, the robot and the environment that are specified separately. eTaSL is chosen as the constraint-based framework, since it offers an easy and compact way to specify a great variety of constraints involving signals coming from different types of sensors. eTaSL uses a reactive velocity-resolved control strategy. It can deal with conflicting constraints by either prioritizing the constraints or by weighting them.

However, it is impossible to add a constraint for each of the cells, due to the large number of cells in the robotic artificial skin. Therefore, as a first contribution, this paper proposes an additional discrete optimization algorithm in order to activate an a-priori selected maximum number of cells. The algorithm selects the appropriate distribution based on the amplitude of each signal and the spatial distribution of the proximity measurements.

The second contribution of this work is a modular methodology by which a human-collaboration task can be specified as an optimization problem subject to constraints. The task is specified as a set of (weighted) constraints. An additional set of constraints involving the input of skin cells is defined such that collisions are avoided. These two sets of constraints are combined to perform the human-collaboration task. Sec. II describes the skin hardware. Sec. III describes the underlying concepts of the eTaSL framework. Sec. IV describes the optimization-based cell selection algorithm. Sec. V explains the reactive control strategy for human-robot collaboration. Sec. VI describes the test setup inspired by an industrial use case. The experimental validation is described in Sec. VII and Sec. VIII contains the discussion and conclusions.

\section{ARtificial Robotic Skin}

The Artificial Robotic Skin, consisting of a modular arrangement of cells that can sense multi-modal tactile stimuli, was developed by Mittendorfer et al. (2015). Multiple sensors are embedded in each cell: (i) a 3D acceleration sensor; (ii) three capacitive force sensors able to measure normal forces; (iii) two temperature sensors; (iv) one optical proximity sensor. A micro controller embedded in each cell collects, filters and sends the most recent signals in packages over the network of sensor cells. Cells are arranged in patches. Patches are flexible and can be mounted on curved surfaces.

The Artificial Robotic Skin comes with a self-calibration procedure (Mittendorfer \& Cheng 2012). The 3D acceleration sensors measure gravity while the robot link is put in different poses. This information is used to determine the transformation from each cell to a reference frame attached to that robot link. The skin used in the experiment is depicted in Fig. 2.

In this work, only the distance sensor is used. Distance sensing is based on a photo-emitter that sends out modulated light, paired with a photo-receiver and filter. The power of reflected light is evaluated and normalised between 0 and 1. While this measurement principle allows to build a very compact cell, the signal is strongly non-linear with respect to the distance and is influenced by the reflecting surface.

The relation between the proximity measure and the distance is calibrated by moving a patch of seven cells on a known trajectory while measuring the proximity to a reflective white surface (Fig. 3). As can be seen in Fig. 3, 


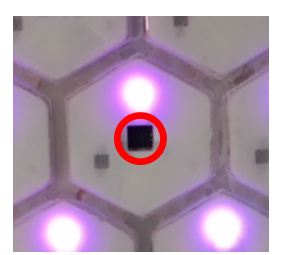

Fig. 2. Close-up of one of the cells of the artificial skin. One edge of the cell measures $1.5 \mathrm{~cm}$. The red circle indicates the distance sensing hardware. Colored leds are used to debug cell connections and to provide visual feedback in robot applications.

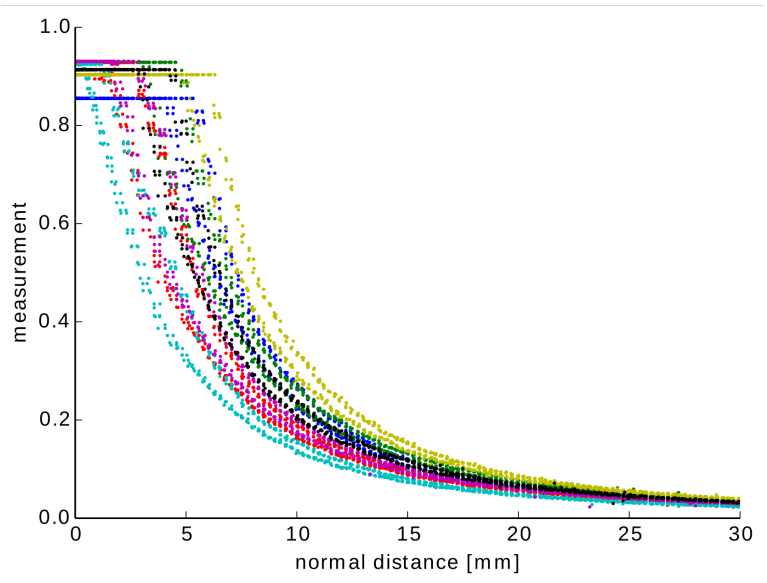

Fig. 3. Proximity sensor characterization. Signals reported for an arrangement of seven cells, showing the high level of uncertainty for small distances.

there is only a noisy non-linear relationship between the proximity measurements and the actual distances. This makes it infeasible to accurately estimate geometrical properties of the perceived objects or even to detect objects beyond 20 - $25 \mathrm{~mm}$. Therefore, distances will not be directly used to formulate collision constraints. Instead, the proximity values will be used to generate a reference velocity, as detailed in Sec. V.

\section{ETASL FRAMEWORK}

eTaSL is a task specification language for reactive control of robot systems. It describes how a robotic system has to move and interact with sensors such as distance, position or force sensors.

eTaSL uses a constraint-based methodology in which a robotic task is specified as an optimization problem subject to constraints. Two priority levels for the constraints are introduced, giving the possibility to specify hard and soft constraints. Besides priorities, the constraints can be also weighted, allowing conflicting constraints.

In contrast to the Task Frame Formalism (Bruyninckx \& De Schutter 1996) or iTaSC (De Schutter et al. 2007), the control is not performed in a frame or using variables of a virtual kinematic tree. Instead, arbitrary expressions are used to describe the constraints. These expressions are specified using expression graphs (Aertbeliën 2012), which encapsulate geometric expressions (e.g. positions, orientations, trajectories), allowing mathematical operation on them.
eTaSL incorporates automatic differentiation, allowing the calculation of Jacobians and higher-order derivatives of these expressions.

A task expression is a function of the following three types of variables: (i) a time variable $t$; (ii) $n_{r}$ robot joint variables $q_{j}$ contained in a vector $\boldsymbol{q} \in \mathbb{R}^{n_{r} \times 1}$; (iii) $n_{f}$ feature variables $\chi_{f, k}$, contained in a vector $\chi_{f} \in \mathbb{R}^{n_{r} \times 1}$, used to facilitate the formulation of the task. While constraints take away motion freedom, feature variables add degrees of freedom to the system.

In this work two types of constraints are introduced:

1) Position constraint. This type of constraint commands a task expression $e_{i}\left(\boldsymbol{q}, \boldsymbol{\chi}_{f}, t\right) \in \mathbb{R}^{1}$, which represents the difference between an expression and a desired target, to evolve towards zero value. This is written as:

$$
e_{i}\left(\boldsymbol{q}, \chi_{f}, t\right) \underset{k_{i}}{\stackrel{w_{i}}{k}} 0
$$

where the term $w_{i}$ is defined to weight the constraint. The velocity-resolved controller enforces the position constraint to evolve towards its target as a first order system with a time constant $1 / k_{i}$; this behavior is described as:

$$
\frac{d}{d t} e_{i}\left(\boldsymbol{q}, \boldsymbol{\chi}_{f}, t\right)=-k_{i} e_{i}\left(\boldsymbol{q}, \boldsymbol{\chi}_{f}, t\right) .
$$

2) Velocity constraint. This type of constraint commands the time derivative of an expression $g_{i}\left(\boldsymbol{q}, \boldsymbol{\chi}_{f}, t\right) \in \mathbb{R}^{1}$ to follow a specific velocity. This is written as:

$$
\text { Time derivative }\left\{g_{i}\left(\boldsymbol{q}, \boldsymbol{\chi}_{f}, t\right)\right\} \stackrel{w_{i}}{\rightarrow} v \text {. }
$$

Note that the behavior of this constraint is influenced only by the weight $w_{i}$. This type of constraint can be transformed into a position constraint, where $k_{i}$ equals zero, in the following form:

$$
\frac{d}{d t}\left(g_{i}\left(\boldsymbol{q}, \boldsymbol{\chi}_{f}, t\right)-v t\right)=0 .
$$

Although in this paper both types of constraints are introduced only as equality constraints, eTaSL also supports the definition of inequality constraints, which are defined in a very similar way to (2) and (4).

Multiple constraints of the above type are used to formulate the robot control as a quadratic programming (QP) optimization problem:

$$
\begin{array}{ll}
\underset{\mathbf{y}}{\operatorname{minimize}} & \mathbf{y}^{T} \mathbf{H y} \\
\text { subject to } & \mathbf{L}_{\mathbf{A}} \leq \mathbf{D y} \leq \mathbf{U}_{\mathbf{A}}
\end{array}
$$

The optimization variable $\mathbf{y}$ is a vector that corresponds to $\left[\begin{array}{ccc}\dot{\boldsymbol{q}} & \dot{\chi}_{f} & \varepsilon\end{array}\right]$ where $\dot{\boldsymbol{q}}$ contains the time derivative of the robot joints, $\dot{\chi}_{f}$ corresponds to a vector with the time derivative of the feature variables, and vector $\varepsilon$ contains the slack variables $\varepsilon_{i}$.

Position constraints (1) are translated into the QP constraints (5b) by expanding the left term in (2) into its partial derivatives; as a result, the equation becomes:

$$
\boldsymbol{J}_{i}\left[\begin{array}{c}
\dot{\boldsymbol{q}} \\
\dot{\boldsymbol{\chi}}_{f}
\end{array}\right]=-k_{i} e_{i}-\frac{\partial e_{i}}{\partial t}+\varepsilon_{i}
$$


In this equation, $k_{i} e_{i}$ corresponds to the feedback control term, while $\frac{\partial e_{i}}{\partial t}$ can be seen as the feed-forward contribution.

For the velocity constraints, the same approach as with the position constraints is used; therefore, expanding the left term in (4) yields:

$$
\boldsymbol{J}_{i}\left[\begin{array}{c}
\dot{\boldsymbol{q}} \\
\dot{\boldsymbol{\chi}}_{f}
\end{array}\right]=v-\frac{\partial g_{i}}{\partial t}+\varepsilon_{i}
$$

The task function Jacobian $\boldsymbol{J}_{i} \in \mathbb{R}^{1 \times\left(n_{r}+n_{f}\right)}$ in (6) and (7) contains the partial derivatives of the task with respect to the robot joint positions and feature variables. This expression is automatically calculated by eTaSL. In case of constraints with a low priority, the slack variable $\varepsilon_{i}$ is added to relax the constraint. For constraints with high priority (hard constraints) this term is left out.

The weights $w_{i}$ specified for each constraint are included in the diagonal of the Hessian matrix $\mathbf{H}$ in (5a). Therefore, the constraints are weighted by weighting the square of their corresponding slack variable $\varepsilon_{i}$. Constraints with weights $w_{i}$ equal to zero do not influence the optimization problem. Therefore, they can be considered as disabled constraints.

The open source solver qpOASIS (Ferreau et al. 2014) is used to solve the optimization problem (5a) and (5b).

\section{OPTIMIZATION OF CELL SELECTION BASED ON SPATIAL DISTRIBUTION AND PROXIMITY}

As hinted before, it is not feasible to impose a collision constraint for each cell, as this will increase the size of the optimization problem drastically. If a fixed subset of cells has to be selected, the most intuitive solution would be to pick cells measuring the highest values. On each cell will be defined a constraint that enforces a velocity applied to the cell center location and directed inward and perpendicular to the cell surface. However, this simplistic policy can result in limit cycles when the skin surface is (almost) parallel with an obstacle. In such a case, a very small motion of the robot can cause the set of selected cells to change drastically, resulting in a radically different control action at each time step.

Therefore, another approach is chosen. The activation value $a_{i}$ of a cell $i$, with $0 \leq a_{i} \leq 1$ corresponds to 1 when an obstacle is touching the cell and corresponds to 0 when there is no obstacle or it is outside the measurement range. Cells with an activation value above a given threshold are called active.

To avoid limit cycles, we seek a policy that selects a subset of the activated cells in such a way that not only the sensor values, but also the subset's spatial distribution is considered. The proposed solution solves an optimization problem that selects a subset of cells that maximizes the activation value of cells and the summed distance along the surface between the selected cells.

As a preprocessing step, a distance map is computed corresponding to the distance along the surface of the skin between each of the cells. Geodesic distance is considered: each patch is unrolled on a flat surface, and the Cartesian distance on this surface is considered. In case of a closeto-cylindrical patch, as for the one encasing the upper arm,
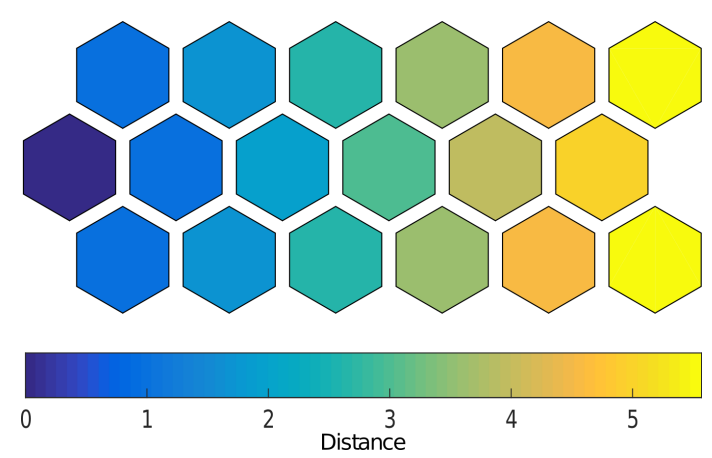

Fig. 4. Distance value, computed from the most left cell to other cells of the patch. Distance is normalized in such a way neighbouring cells have a distance of 1 .

geodesic distance on a cylinder is used. The distance map from one cell to all other cells of the same patch is illustrated in Fig. 4. The patch in Fig. 4 is mounted on the last link of the robot (the one touched in Fig. 6).

The optimization problem is then formulated as follows: Given $\mathcal{A}$ the set of active cells, with cardinality $N_{a} ; N_{s}$ the number of cells to select; $d_{i, j}>0$ as the distance between cells, find the subset $\mathcal{S} \subseteq \mathcal{A}$, with $\operatorname{card}(S)=N_{s}$, that satisfies the following optimization problem:

$$
\begin{aligned}
& \max _{\mathcal{S}} \nu \sum_{i \in \mathcal{S}} a_{i}+\sum_{\substack{i, j \in \mathcal{S} \\
j>i}} d_{i, j} \\
& \text { s.t. } \mathcal{S} \subseteq \mathcal{A}, \operatorname{card}(S)=N_{s},
\end{aligned}
$$

where $\nu$ is a trade-off factor between highest activation and spatial distribution.

This problem can be re-formulated as a binary linear programming problem, in the form:

$$
\begin{aligned}
\max _{\mathbf{x}} & \mathbf{c}^{T} \mathbf{x} \\
\text { s.t. } & \mathbf{x} \in\{0,1\}^{N} \\
& \mathbf{A}_{e} \mathbf{x}=\mathbf{b}_{e} \\
& \mathbf{A}_{i} \mathbf{x} \leq \mathbf{b}_{i} .
\end{aligned}
$$

The state vector $\mathbf{x}$ contains a binary variable for each activated cell, plus a variable for each possible pair of cells. The number of pairs $N_{c}$ is given by $1 / 2 N_{a}\left(N_{a}-1\right)$. $N=N_{a}+N_{c}$ corresponds to the size of $\mathbf{x}$.

The cost vector $\mathbf{c}$ can be formalized as:

$$
\begin{aligned}
\mathbf{c}=[ & {\left[\nu \cdot a_{1} \cdots \nu \cdot a_{N_{a}},\right.} \\
& \left.\quad d_{1,2} \ldots d_{1, N_{a}}, d_{2,3} \ldots d_{2, N_{a}}, \ldots d_{N_{a}-1, N_{a}}\right]^{T},
\end{aligned}
$$

where the second part contains $N_{c}$ elements. The equality constraints take care that exactly $N_{s}$ cells and $N_{c}$ cell couples are selected:

$$
\mathbf{A}_{e}=\left[\begin{array}{ll}
\mathbf{1}_{1 \times N_{a}} & \mathbf{0}_{1 \times N_{c}} \\
\mathbf{0}_{1 \times N_{a}} & \mathbf{1}_{1 \times N_{c}}
\end{array}\right], \mathbf{b}_{e}=\left[\begin{array}{c}
N_{s} \\
N_{s} \frac{\left(N_{s}-1\right)}{2}
\end{array}\right] .
$$

The inequality constraints enforce the relation between the selected cells (first part of $\mathbf{x}$ ) and the pairs (second part of $\mathbf{x}$ ), and impose the following $N_{c}$ inequalities for all $i$ and $j$ : 


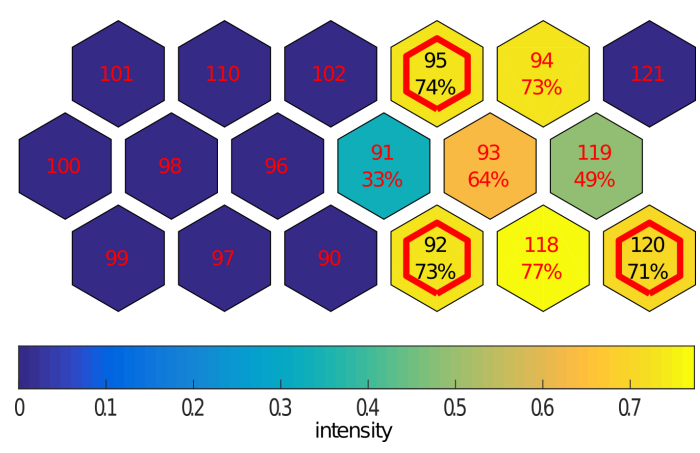

Fig. 5. Simulated activation of cells. Eight cells are activated and three are selected (red hexagon overlay). Each cell has an ID number and the activation value in percentage, and is colored according to the activation value. It can be seen that the most active cell (118) is not selected, in favour of some of its neigbours.

$$
\begin{aligned}
& x_{i}+x_{j}-x_{z} \leq 1, \\
& \text { with } \\
& \qquad \begin{array}{l}
1 \leq i<j \leq N_{a}, \\
\qquad z=i \cdot N_{a}-i \cdot \frac{i-1}{2}+j-i
\end{array}
\end{aligned}
$$

To illustrate the structure of the matrix, the inequalities in matrix form for $N_{a}=4$ are given below:

$$
\mathbf{A}_{i}=\left[\begin{array}{ccccc}
1 & 1 & 0 & 0 & \\
1 & 0 & 1 & 0 & \\
1 & 0 & 0 & 1 & -\mathbf{I}_{N_{c} \times N_{c}} \\
0 & 1 & 1 & 0 & \\
0 & 1 & 0 & 1 & \\
0 & 0 & 1 & 1 &
\end{array}\right], \quad \mathbf{B}_{i}=\mathbf{1}_{N_{c} \times 1}
$$

An optimization problem is solved for every patch independently. Time execution varies depending on the number of active cells, and can be slower than the frequency at which cell measurement data is gathered by the interface. The index of selected cells is maintained until a new solution is computed, while the values of the selected cells are updated every time new data is made available by the cell interface. The optimization problem is implemented with the qp_solve library (Berkelaar et al. 2004).

An example solution of the described problem is reported in Fig. 5, where the activation of eight cells is depicted, from which three are selected.

\section{REACTIVE ROBOT MOTION CONTROL}

This section presents four motion control laws, specified in the constraint-based methodology described in Sec. III. These constraints allow human-robot collaboration in a robotic task in which the end effector moves between two points following a nominal trajectory.

\section{A. Task execution: Trajectory following}

\section{Constraint 1: move along path}

The trajectory followed by the end effector to perform the desired task is composed of a nominal path from an initial position, towards a desired target; and a corresponding

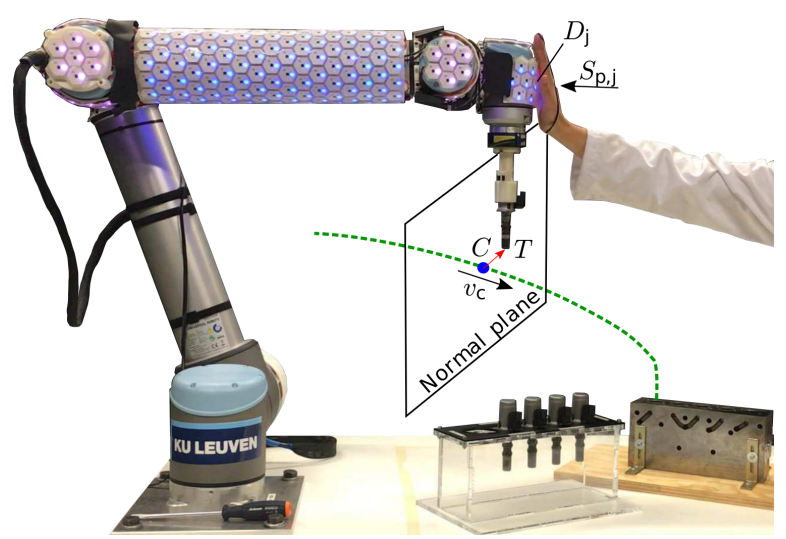

Fig. 6. Robot behavior along the trajectory. The green dashed line corresponds to a nominal path.

orientation of the end effector along the path. This orientation is calculated by a linear interpolation between the orientation of the end effector at the initial point, and its desired orientation at the target.

To describe the motion of the end effector along the path, first a position vector $\boldsymbol{C}\left(\chi_{f, 1}\right) \in \mathbb{R}^{3 \times 1}$ is defined on the nominal path (see Fig. 6). Note that $C$ is defined in function of the path coordinate $\chi_{f, 1}$, which is specified as feature variable in eTaSL.

A trapezoidal velocity profile $v_{c}$ is designed in function of this path coordinate $\chi_{f, 1}:(i)$ at the starting section of the path, $v_{c}$ rises at a constant rate $a_{\max }$ until reaching $v_{\max }$; (ii) in the middle section, $v_{c}$ keeps a value of $v_{\max }$; (iii) during the final section, $v_{c}$ decreases at a constant rate $-a_{\max }$ until it reaches zero velocity at the specific target. The motion of point $\boldsymbol{C}\left(\chi_{f, 1}\right)$ is constrained to follow the velocity profile $v_{c}$ using a velocity constraint (see (3) and (4)):

$$
g_{1}:=\chi_{f, 1} \quad \text { and } v:=v_{c} .
$$

If a constraint with higher weight $w_{i}$ is conflicting with $g_{1}$ (e.g. a object detected near to the robot) the velocity of $\boldsymbol{C}$ can still be adapted because this is a soft constraint. This velocity can decrease towards zero or even take negative values, causing the robot to move backwards.

\section{Constraint 2: remain on path}

To command the motion of the robot end effector, a position vector $\boldsymbol{T}(\mathbf{q}) \in \mathbb{R}^{3 \times 1}$ is defined in the tool as depicted in Fig. 6. To attach $\boldsymbol{T}(\mathbf{q})$ to $\boldsymbol{C}\left(\chi_{f, 1}\right)$, a position constraint (see (1) and (2)) is specified:

$$
e_{2}:=\boldsymbol{T}(\mathbf{q})-\boldsymbol{C}\left(\chi_{f, 1}\right)
$$

In this case $\boldsymbol{e}_{\mathbf{2}} \in \mathbb{R}^{3 \times 1}$. Hence, each element of $\boldsymbol{e}_{\mathbf{2}}$ is included as a separate constraint in $(5 b)$.

\section{B. Reactive behavior: Collision avoidance}

\section{Constraint 3: avoid collisions}

The discontinuous change between active/non-active cell $j$ can cause instabilities/limit cycles.

To improve the behavior caused by the transition between active and non-active cells, an improved selection procedure 
is proposed based on the weight $w_{3, j}$ assigned to each cell: (i) all the weights $w_{3, j}$ are initialized in zero; (ii) after a cell is selected by the optimization problem (Sec. IV), its weight $w_{3, j}$ rises proportionally to the time it has been selected. If a cell is rejected, $w_{3, j}$ decreases at the same rate; (iii) then, a number up to $N_{s}$ cells with the highest nonzero weights are selected. (iv) the weights $w_{3, j}$ are normalized dividing their value by the number of nonzero weights.

Velocity constraints are defined using the selected cells $j$ with their corresponding weight $w_{3, j}$. The control law is designed based on a damping force controller described in (Bruyninckx \& De Schutter 1996). While in the damping force controller the correcting velocities are function of the force errors, in the proposed method these velocities are proportional to the sensed proximity signals:

$$
v_{p, j}:=C_{p} S_{p, j}
$$

where $C_{p}$ is a proportional constant.

The motion of the position vector $\boldsymbol{D}_{\boldsymbol{j}}(\mathbf{q}) \in \mathbb{R}^{3 \times 1}$ located in the center of a selected cell is constrained to follow a velocity $v_{p, j}$ directed inward and perpendicular to the cell surface (i.e. $v_{p}$ is projected on $-\hat{\boldsymbol{n}}_{\boldsymbol{j}}$ ). In eTaSL, this is implemented as a velocity constraint (see (3) and (4)):

$$
\boldsymbol{g}_{\mathbf{3}, \boldsymbol{j}}:=\boldsymbol{D}_{\boldsymbol{j}}(\mathbf{q}) \quad \text { with } \boldsymbol{v}_{\boldsymbol{j}}:=v_{p, j} \cdot \hat{\boldsymbol{n}}_{\boldsymbol{j}}
$$

Similarly to (15), $\boldsymbol{g}_{3, j} \in \mathbb{R}^{3 \times 1}$ and each of its elements is included separately in $(5 \mathrm{~b})$.

\section{Constraint 4: remain on normal plane}

A soft position constraint (see (1) and (2)) is set to restrict $\boldsymbol{T}(\mathbf{q})$ to the normal plane of the trajectory at point $\boldsymbol{C}\left(\chi_{f, 1}\right)$. The desired behavior of $e_{4}$ is given by:

$$
e_{4}:=\left(\boldsymbol{T}(\mathbf{q})-\boldsymbol{C}\left(\chi_{f, 1}\right)\right) \cdot \hat{\boldsymbol{t}}_{t r a j}
$$

where $\hat{t}_{t r a j}$ is the tangent vector of the trajectory at point $\boldsymbol{C}\left(\chi_{f, 1}\right)$ (see Fig. 6).

\section{USE CASE}

This section describes a use case involving human-robot collaboration inspired by an industrial application.

The complete manual assembly operation consists of retrieving five solenoids from a rack. Subsequently, the solenoids are inserted and fixed to a main hub. Each solenoid is fixed to the hub by two screws positioned at opposite sides of its body. The workstation for the application is shown in Fig. 1. The assembly sequence for one solenoid is summarized in table I.

The high load on the insertion operation is strenuous for the human operator when executed repetitively. This, combined with the relatively low level of dexterity required, make the solenoid manipulation suitable for robotic automation. On the other hand, the screw handling operations require a higher level of dexterity and precision. The small size of the handled objects presents a bigger challenge to grasp and align them. Moreover, there can be a slight misalignment between the screw holes of the solenoid and their counterparts in the hub. During a manual screw handling, these issues could be easily resolved by an operator.

\begin{tabular}{|c|c|c|c|c|}
\hline Object & Tools & Step & Operation & Requirements \\
\hline solenoid & gripper & $\begin{array}{l}1 \\
2 \\
3 \\
4\end{array}$ & $\begin{array}{l}\text { retrieve } \\
\text { transport } \\
\text { align } \\
\text { insert }\end{array}$ & $\begin{array}{c}\text { grip } 3 \mathrm{~cm} \\
\text { carry } 260 \mathrm{~g} \\
<5 \mathrm{~mm} \text { and }<1^{\circ} \\
\text { push } 100 \mathrm{~N}\end{array}$ \\
\hline \multirow[t]{2}{*}{ screw x2 } & human hand & $\begin{array}{l}5 \\
6 \\
7\end{array}$ & $\begin{array}{l}\text { take } \\
\text { transport } \\
\text { align }\end{array}$ & $\begin{array}{l}\text { grip } 5 \mathrm{~mm} \\
\text { carry } 4 \mathrm{~g} \\
<0.5 \mathrm{~mm}\end{array}$ \\
\hline & screw driver & 8 & insert & turn $>10$ times \\
\hline
\end{tabular}

TABLE I

MANUAL ASSEMBLy SEQUENCE FOR ONE SOLENOID.

Therefore, to reduce assembling time and increase production efficiency for both human and robot, we propose to perform the manual and automated operations simultaneously. In the proposed scenario, as soon as the robot finishes the solenoid insertion, the operator starts to insert the screws to fix the inserted solenoid to the hub. While the operator performs these operations, the robot continues with the subsequent tasks: retrieve, transport and align the next solenoid. If the robot approach to insert a solenoid while the operator still is in the workspace performing his task, the operator can interrupt the robot motion by placing his hand in front of the robot's end effector (see Fig. 1). Once the operator finishes his task leaving the workspace, the robot continues its motion to insert the next solenoid.

\section{EXPERIMENTAL RESULTS}

This section first describes how to configure the control parameters for the collaborative controller. Secondly, an isolated experiment is described where the robot avoids an obstacle. Finally, the results of a human-robot collaboration in a laboratory mock-up of an industrial environment are presented.

\section{A. Tuning Control Parameters}

The constraint parameters are summarized in table II

TABLE II

CONTROL PARAMETERS.

\begin{tabular}{lccl}
\hline Constraint & Parameter & Value & Unit \\
\hline \multirow{3}{*}{ 1: move along path } & $v_{\max }$ for $v_{c}$ & 0.22 & $\mathrm{~m} / \mathrm{s}$ \\
& $a_{\max }$ for $v_{c}$ & 0.5 & $\mathrm{~m} / \mathrm{s}^{2}$ \\
& $k_{1}$ & 0 & $\mathrm{~s}^{-1}$ \\
& $w_{1}$ & 0.01 & \\
\hline 2: remain on path & $k_{2}$ & 0.55 & \multirow{2}{*}{$\mathrm{s}^{-1}$} \\
& $w_{2}$ & 0.5 & \\
\hline \multirow{2}{*}{ 3: avoid collisions } & $C_{p}$ & 0.1 & $\mathrm{~m} / \mathrm{s}$ \\
& $k_{3, j}$ & 0 & $\mathrm{~s}^{-1}$ \\
\hline \multirow{2}{*}{ 4: remain on normal plane } & $w_{3, j}$ & $0-1$ & \\
\hline
\end{tabular}

The behavior of a robotic application is influenced by the relation between the constraint weights $w_{i}$, in particular for 
this application: (i) The robot motion along the path must react fast enough in case a potential collision is perceived. To this end, $w_{1}$ is set with a small value. (ii) Constraint 3 determines the reaction of the robot when a collision is sensed by the artificial skin. The gain $C_{p}$ is set to assure that the maximum reacting velocity takes a value of $0.1 \mathrm{~m} / \mathrm{s}$. The weights $w_{3, j}$ of each selected cell are set to have a maximum combined value of 1 . The rate at which they increase/decrease is tuned to have a fast response to avoid collisions. (iii) The relation between constraints 2 and 3 influences the robot behavior when deviating from the nominal path (see Fig. 6). Parameters $w_{2}$ and $k_{2}$ are set to let the robot deviate from the nominal path if a collision is detected, while driving the robot back to the nominal path fast enough if a collision is not perceived. (iv) Constraint 4 does not affect directly the robot behavior.

\section{B. Circumventing an obstacle}

In this isolated experiment, the robot must follow a nominal path (see Fig. 7). An object, with a convex shape, is placed close enough to the nominal path to interfere with the end effector motion. The robot avoids collision while circumventing the obstacle based on the readings of the proximity sensors. Once the obstacle is avoided the end effector continues its motion towards the target.

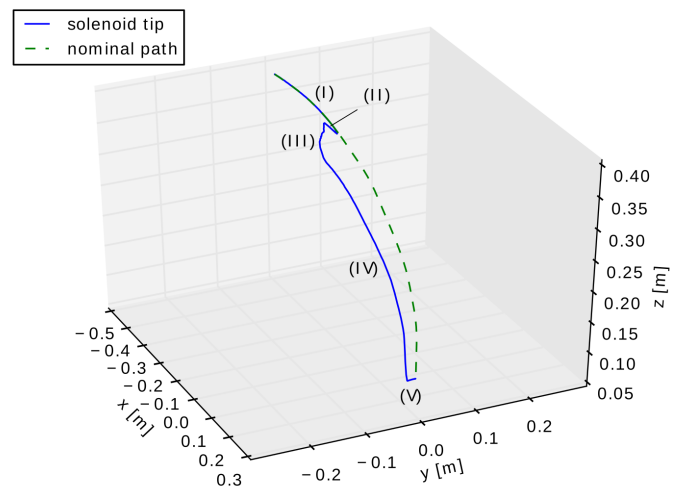

Fig. 7. 3D path followed by a solenoid tip to circumvent an obstacle located along a nominal path.

This task can be divided into five phases (figs. 7 and 8):

(I) In the first phase the robot follows the trajectory with a trapezoidal velocity profile. Constraint 3 (table II) is disabled, because there are no selected cells (the associated weights $w_{3, j}$ are equal to zero).

(II) As soon as the cells of the artificial skin sense an object, the signals $S_{p, j}$ increase rapidly, hence the associated weights $w_{3, j}$ start to increase. Consequently, the velocity along the path reverses its sign, resulting in the end effector moving backwards along the path in order to avoid collision with the obstacle. At the same time the end effector starts to deviate from the nominal path.

(III) In this phase the end effector is far enough from the obstacle. The resulting behavior is a trade-off between constraint 1 pushing the robot further along the path (i.e. trying to increase $\chi_{f, 1}$ ), constraint 2 trying to keep the robot on the trajectory and constraints 3 trying to avoid collisions. The proximity signals $S_{p, j}$ are still active with a lower value compared with the previous phase.

(IV) The forth phase starts when the end effector finishes circumventing the obstacle. There are no more signals $S_{p, j}$ reported from the skin, the weights of the cells start to decrease until they reach zero. The distance between the solenoid tip and the nominal path decreases, leading the end effector towards the nominal path.

(V) In the final phase the path coordinate $\chi_{f, 1}$ has reached its maximum value leading $C$ to the end of the path. However, the tool point $\boldsymbol{T}$ (solenoid tip) has not yet arrived at the desired target. Immediately after the weights $w_{3, j}$ reach zero value, the distance between the solenoid tip and the nominal path decreases according to a first order system parameterized by the control constant $k_{2}$. As a result, $\boldsymbol{T}$ evolves towards the target.

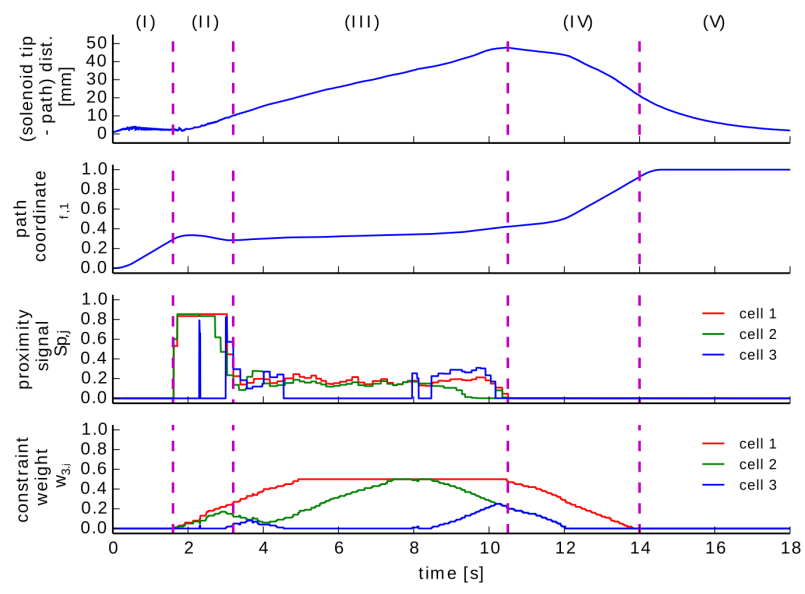

Fig. 8. Experimental result of robotic end-effector circumventing an obstacle located along a nominal path. The solenoid tip deviates $50 \mathrm{~mm}$ from a nominal path to avoid collisions

\section{Use Case}

Experimental results from a full sequence of the use case, described in Sec. VI, are presented here.

In Fig. 9, the left column presents the results of the first solenoid insertion; in this case there is no human intervention, therefore, the path coordinate follows the trapezoidal velocity profile $v_{c}$. In the insertion of the four subsequent solenoids, the operator interrupts the robot motion by placing his hand against the end effector to interrupt its motion. After finishing his task, the operator removes his hand, letting the robot continues its task.

In contrast to the previous experiment, in this case the velocities computed from the proximity signals lead the robot to avoid collisions going backwards in a first phase 


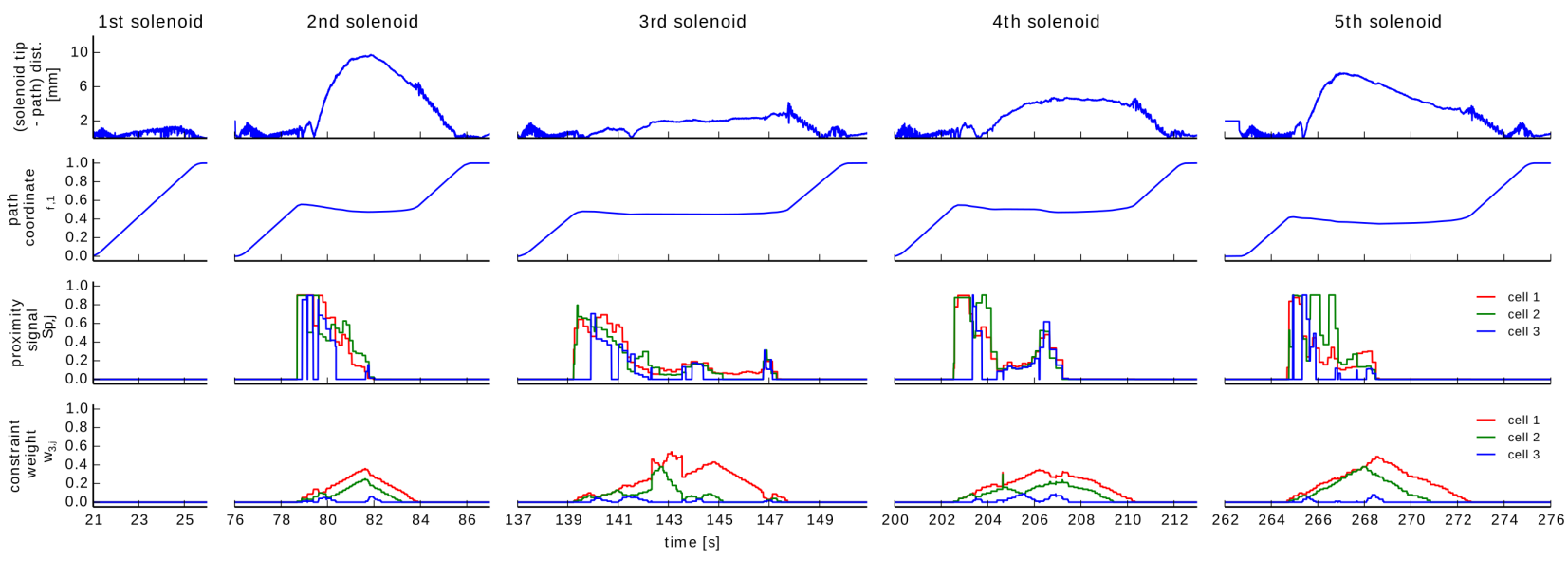

Fig. 9. Experimental results of a human-robot collaboration use case inspired by an industrial scenario: a robotic manipulator transports five solenoids sequentially from an initial pose $\left(\chi_{f, 1}=0\right)$ towards a target pose $\left(\chi_{f, 1}=1\right)$, in which the solenoid is aligned above a corresponding hole. The operator interrupts the robot motion to finish the fixing operation by placing his hand in front of the artificial skin. As a result, proximity signals $S_{p, j}$ are activated increasing the constraint weights $w_{3, j}$. Only solenoids transport phases are shown.

and then maintaining its position. In Fig. 9 it is shown that the maximum deviation of the end effector from the path is $10 \mathrm{~mm}$, which is not enough to circumvent the operator's hand. The path coordinate presents the following behavior: $(i)$ first $\chi_{f, 1}$ has a slight decrement immediately after the proximity signals are activated, therefore, the end effector moves backwards along the path; (ii) subsequently $\chi_{f, 1}$ maintains its value until the operator removes his hand from the path; (iii) at this stage the weights $w_{3, j}$ decrease until they reach zero, deactivating constraints 3; (iv) finally constraints 1 and 2 drive the end effector towards the desired target.

\section{CONCLUSIONS}

This paper discusses a new framework for the specification of robotic tasks where human-robot collaboration is involved. A strategy is presented to deal with a large number of proximity signals and selecting the appropriate input to a constraint-based reactive controller.

The cell selection algorithm showed to be an effective method to deal with signals coming from 373 cells embedded in an artificial robotic skin. A discrete optimization algorithm provides this selection by considering a threshold between the intensity reported by each cell and the spatial distribution of the activated cells. This strategy was validated both in simulations and in real experiments.

An interaction modeled by four conflicting constraints results in a task execution scenario where a robot advances along a nominal path, while offering the ability to avoid collisions by stopping, moving backwards along the path or deviating from a nominal path to circumvent obstacles. This behavior is independent of the end effector orientation.

A first experiment demonstrates a case where a robot is able to circumvent a convex obstacle, deviating $50 \mathrm{~mm}$ from a nominal path, to later reach a desired target. A second experiment tests the framework in a use case inspired by an industrial application. The robot is commanded to insert five solenoids in a main hub, while the human interrupts the robot motion to fix the solenoids.

In the current framework, only collisions with the surfaces covered by the artificial skin can be avoided, however, it is not possible to perceive and avoid collisions with the tool or the solenoid. Future work will extend our methods to combine other types of sensors with an extended perception range, such as tracking devices and point cloud sensors.

\section{ACKNOWLEDGMENT}

The Robotics Research Group is a university core lab of Flanders Make.

All authors gratefully acknowledge the financial support by: The European Communitys Seventh Framework Programme project Factory-in-a-day(FP7-609206), Flanders Make Project Yves and Technical University of Munich (TUM) for providing the artificial skin.

\section{REFERENCES}

Aertbeliën, E. (2012), 'Expression graph library'. Available from https://github.com/eaertbel/expressiongraph [Accessed 15 February 2018].

Aertbeliën, E. (2016), 'The etasl task specification language'. Available from https://people.mech.kuleuven.be/ eaertbel/etasl [Accessed 27 January 2018].

Aertbeliën, E. \& De Schutter, J. (2014), eTaSL/eTC: A constraintbased task specification language and robot controller using expression graphs, in 'IEEE/RSJ International Conference on Intelligent Robots and Systems', pp. 1540-1546.

Albini, A., Denei, S. \& Cannata, G. (2017), Enabling natural human-robot physical interaction using a robotic skin feedback and a prioritized tasks robot control architecture, in 'IEEERAS 17th International Conference on Humanoid Robotics (Humanoids)', pp. 99-106.

Bélanger-Barrete, M. (2015), Collaborative Robot ebook, sixth edn, ROBOTIQ.

Berkelaar, M., Eikland, K. \& Notebaert, P. (2004), 'lp_solve 5.5, open source (mixed-integer) linear programming system'. Available from http:// lpsolve. sourceforge.net / 5 . 5/ [Accessed 10 February 2018].

Bruyninckx, H. \& De Schutter, J. (1996), 'Specification of forcecontrolled actions in the "Task Frame Formalism": A synthesis', Vol. 12 (No. 5), 581-589. 
Buizza Avanzini, G., Ceriani, N. M., Zanchettin, A. M., Rocco, P. \& Bascetta, L. (2014), 'Safety control of industrial robots based on a distributed distance sensor', IEEE Transactions on Control Systems Technology Vol. 22 (No. 6), 2127-2140.

De Schutter, J., De Laet, T., Rutgeerts, J., Decré, W., Smits, R., Aertbeliën, E., Claes, K. \& Bruyninckx, H. (2007), 'Constraintbased task specification and estimation for sensor-based robot systems in the presence of geometric uncertainty', The International Journal of Robotics Research Vol. 26 (No. 5), 433-455.

Dumonteil, G., Devy, M., Confetti, A. \& Sidobre, D. (2015), Reactive planning on a collaborative robot for industrial applications, in '12th International Conference on Informatics in Control, Automation and Robotics (ICINCO)', pp. 450-457.

Factory in a Day (2014). Available from http://www. factory-in-a-day.eu/ [Accessed 27 January 2018].

Ferreau, H., Kirches, C., Potschka, A., Bock, H. \& Diehl, M. (2014), 'qpOASES: A parametric active-set algorithm for quadratic programming', Mathematical Programming Computation Vol. 6 (No. 4), 327-363.

Haddadin, S., Albu-Schaffer, A., De Luca, A. \& Hirzinger, G. (2018), Collision detection and reaction: A contribution to safe physical human-robot interaction, in 'IEEE/RSJ International Conference on Intelligent Robots and Systems', pp. 3356-3363.

Hornung, A., Wurm, K. M., Bennewitz, M., Stachniss, C. \& Burgard, W. (2013), 'OctoMap: An efficient probabilistic 3D mapping framework based on octrees', Autonomous Robots .

Luo, R., Hayne, R. \& Berenson, D. (2017), 'Unsupervised early prediction of human reaching for human-robot collaboration in shared workspaces', Autonomous Robots pp. 1-18.

Mansard, N., Stasse, O., Evrard, P. \& Kheddar, A. (2009), A versatile generalized inverted kinematics implementation for collaborative working humanoid robots: The Stack of Tasks, in 'IEEE International Conference on Advanced Robotics', pp. 1-6.

Mittendorfer, P. \& Cheng, G. (2012), 3d surface reconstruction for robotic body parts with artificial skins, in 'IEEE/RSJ International Conference on Intelligent Robots and Systems', IEEE, pp. 4505-4510.

Mittendorfer, P., Yoshida, E. \& Cheng, G. (2015), 'Realizing wholebody tactile interactions with a self-organizing, multi-modal artificial skin on a humanoid robot', Advanced Robotics Vol. 20 (No. 1), 51-67.

Sadrfaridpour, B. \& Wang, Y. (2018), 'Collaborative assembly in hybrid manufacturing cells: An integrated framework for humanrobot interaction', IEEE Transactions on Automation Science and Engineering Vol. 15 (No. 3), 1178-1192.

Shin, H., Seo, K. \& Rhim, S. (2018), Allowable maximum safe velocity control based on human-robot distance for collaborative robot, in '15th International Conference on Ubiquitous Robots (UR)', pp. 401-405.

Zanchettin, A. M., Ceriani, N. M., Rocco, P., Ding, H. \& Matthias, B. (2016), 'Safety in human-robot collaborative manufacturing environments: Metrics and control', IEEE Transactions on Automation Science and Engineering Vol. 13 (No. 2), 882-893.

Zeestraten, M. J. A., Pereira, A., Althoff, M. \& Calinon, S. (2016), Online motion synthesis with minimal intervention control and formal safety guarantees, in '2016 IEEE International Conference on Systems, Man, and Cybernetics (SMC)', pp. 002116-002121. 